\title{
Index
}

Acapulco 25, 103

Aceh(nese) $37,63,66-8,70-5,110,121,127$, $132,139,146,152,156-7$

Africa 42,114

Agastya $5^{1}$

Age of Commerce $11,117,120-3,125^{-8,} 130$, $132,134,137-8,145$

Ahern, George 21

Airlangga, Rake $\quad 50,60$

Alaska 64

Alcina, Francisco Ignacio $\quad 79,83-4,86-7$, 91-2

Aldrovandi, Ulisse $\quad 97$

Algeria 44

Alpers, Svetlana 181

Amangkurat II 191, 194-5

America(n)(s) 27-30, 42, 44, 121, 139, 165, 192

Americas 25, 38-9, 8 o see also America,

New Spain, United States, USA

Amoy 145, 147

Amsterdam 182-7, 190-1, 200

Andaman 71

Andaya, Barbara $\quad 153,156$

Anderson, Benedict $\quad 176$

Angkor 7, 40, 126, 130-1

Anglo-Dutch War 160

animals $\quad 1,15^{-6}, 23,30-2,38,40,42,44-5$, $96,99,100,102-3,106,110-2,190,200$

Annam $\quad 116$

Antwerp 101

Arabia(n)/ Arabic/ Arab(s) 38-9, 42-3, 66, $82,99,110,121-2,150$

Arafura 135

Arcimboldo, Giuseppe $\quad$ 96, 99

Aristotle 102

Arung Palakka 137, 139, 143

Asahan 73

Asia Minor 105

Australia(n) $36,42-3,45,77,100,150$

Ayutthaya 121, 153-4

Bajo 154

Bali(nese) $36,54,65-6,124,135,164,167$, 169-70, 180, 191

Balitung, Rake $\quad 48,5^{0}, 55^{-7}$
Banda Aceh $\quad 66-7,73-5$

Banda(nese) $\quad 101,135,138-9,162$

Bangka 154

Banjarese $\quad 163$

Banjarmasin 135, 152

Banka 106

Bankoff, Greg 8-9

Bantam 146

Banten(ese) $137,146,151,158,161,163,186-7$

Banyuwangi 193

Barus $\quad 67-8,71,73,115$

Bastin, John 62

Basuki Abdullah, R. 194

Batak(s) 37, 69, 73, 157

Batangas 82

Batavia $38,103-4,147,151-4,160,181-6$, 188-90, 197

Bätschmann, Oskar 99

Battambang 40

Baud, J.C. 196

Bay of Bengal 106

Beeckman, Andries $\quad 185$

Bellini, Giovanni $\quad 96,99$

Bengkulu 64, 66-7

Berry, Brian 136

Betawi 166, 180

Bik, Adrianus Johannes 192, 195

Billiton 106

Blussé, Leonard $\quad$ 162, 191

Boeke, J.H. 5

Bohol 82, 84, 91-2

Bone $135,137,139,142-3,145-8,177$

Bontius, Jacobus 103

Boomgaard, Peter $\quad 1,2,8-10,13,32,45,62,65$, $68,78,83,86,88,95,100,120,132-3,140$, 167,183

Borneo $37,87,92,106,109,112,115-8,134$, $138,154,157,163,165$

West 154, 165 see also Kalimantan

Borobodur 2, 35

Brandes, J.E. (Jan) 189, 192

Brantas (river) 123

Braudel, Fernand $\quad 2-4,6-7,106,120,131,135$, 137, 167, 181, 201

British Singapore 121 
British/ Britain $\quad 6,42,44-5,121,128,147$

Bromo, Mt. 6o-1

Brunei $82,151-2,154$

Buddha 59, 188-9

Buddhism/ Buddhist $\quad 7-9,10,45,5^{0-2}$, $56,60-1,73-4,108-9,114,121-3$, 126-7, 190

Theravada $\quad 7,121-2,126-7$

Bugis $137,139,142-3,145,148,15^{2}, 156$

Buitenzorg (Bogor) 189, 196

Bulbeck, David 140-1

Burke, Peter 12,181

Burma $6,7,33-5,39-40,42,44,106,122$, $126-7,129-31,154,174$

California 63,72

Cambay 115

Cambodia $34,37,39,107,115,117,129,138-9$, $158,163-5$

Camel, Georg Josef 103

Canton 105, 107

Cape Town 188,190

Cardeel, Lucas $\quad 186-7$

Caribbean 43, 49, 102

Carletti, Francesco 87

Catherine of Austria 101

Catholicism/ Catholic(s) $\quad 80-1,90,103,182$

Cavite 82

Cebu 24, 82, 84, 91, 94, 152

Celebes see Sulawesi

Central/ Inner Asia(n) 35, 39, 131, 187

Cephas, Kassian 197

Ceylon 115, 153-4 see also Sri Lanka

Cham 5, 40, 75 see also Champa

Champa $37,40,117,122,150$

Chang Hu 112

Chantaburi 154-5, 164

Chao Ju Kua 40, 114-6

China 9, 11, 23, 29, 32-4, 36-8, 41-2, 44-5, $74-5,80,91,100,105-10,112-4,116-7$, $121-3,126-7,129,131,134,136,139,145$, $147,150-1,158,162-4,186$

Chinese $5,8,11-2,24,33-4,36-7,40-1,55$, $74-5,82,101-2,104-13,115-8,120,122$, $126,129-30,134,138-9,142,145^{-6}$, $148-65,182,185-8,192,197-8$

Chirino, Pedro 79

Chola kingdom $\quad$ 122, 126

Chou Ch'u-fei 114
Chou Ta-kuan see Zhou Daguan

Christian(s)/ Christianity 41, 80, 90-1, 94, 99, 106, 144, 199

Cilacap 69,76

Clarence-Smith, William 9

clientelism $\quad 8,12-3,167-8,170,172-5,178-9$

Cnoll, Pieter $\quad 185,191$

Cochinchina $152,156,162$

Colombijn, Freek 62

commerce $8,11,102,110,112,114,117-8$, $120-4,127,129-132,134-9,145,148$, 157-9, 183

Company see voc

Cordilleras 26

Coromandel 122, 139, 157-8, 188

Coyett, Frederik $\quad$ 188-9

Crouch, Harold 176

Cummings, William 137, 145

Cuyp, Aelbert $\quad 184-5$

Daendels, H.W. $\quad$ 193, 196

Danes, Danish 138, 157

Dayak 166, 180

Dayak Adat Defence Command 166-7

De Bevoise, Ken 79

democracy $\quad 6,12,178-9$

demography/demographic $1,8,10,11$, 78-81, 83, 86, 90, 93, 95, 100, 121, 129, $131-2,183$

Dewey, Alice 149

Dieng $47,51,54-7,123$

Dioscorides 102

Diponegoro War 193 see also Java War

Diponegoro, Pangeran 194-6

Dobbin, Christine 62

donkeys $32,34-5,38,40,43-5$

Douglas, Mary 85

Dubbels, Hendrick Jacobsz $\quad 183-4$

Dutch 11, 25, 38-9, 42-3, 64-5, 69, 76, 90, $102-4,121,127-8,136-9,141-3,146-8$, 151-3, 159-61, 171-5, 181-92, 194-201 see also Holland, Netherlands

Dutch East Indies Company (voc) see voc

earthquake $7,9-10,28,31,46-7,50,55$, $62-77,131$

East Timor 44,70

Eckhout, Albert $\quad 185$

Egypt 105 


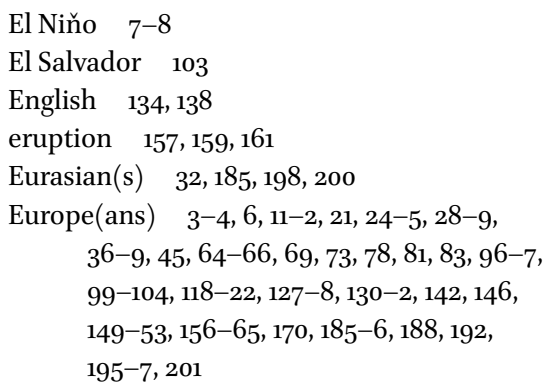

Fang Ch'ien-li 111

fauna $23,97,103,115,192$

Feith, Herbert $\quad 173,175$

Fernández-Armesto, Felipe $\quad 181$

Filipinos 24, 26, 30, 81

flora $23,97,103,188,192,197$

Flores 65, 135

Flores, Jorge 107

Foreman, John 44

forest(ry) $\quad 1-2,6,8,9,15-6,18,20-31,96,100$, $102-4,112,117,122,126,145,148,156,163$, $183,193,196-7,199$

France $\quad 44,130,191$

French 2, 5, 28, 42, 44, 103, 121

Fujian(ese) $\quad 37,117$

Fukien 114

Funan 106, 126

Galunggang 75

Gama, Vasco da 101

Ganeśa $\quad 51$

Geertz, Clifford 149

Gelman Taylor, Jean $\quad 12$

Genoa 101, 144

Goa 158

Godley, David 131

Golconda 146

Golkar 176, 178

Gould, James 62

Gowa(n) 137, 139-40, 143-5

Gowa-Talloq 137-8, 143-5

Gramberg, Jan Simon Gerardus 192-3

Greenland 131

Gresik 150, 198

Guangdong 105, 117, 186

Guangxi 105

Guatemala 103

Guided Democracy $\quad 173-4$
Gulf of Siam 106, 155, 162-3

Gunung Agung, Mt. 65

Hadhrami 43

Hamengkubuwono VII 197

Hamilton, Alexander 66

Han (era) 33, 41, 105, 107

Hanoi 105,107

Hatien 154, 162

Henley, David ～11, 86, 88, 93, 95, 116

Hillen, W.P. $\quad 198$

Himalayas 33

Hindu 10, 51, 53-4, 56-8, 61, 112, 123, 126

Hokkaido 70-1

Holland $\quad 182-7,189-90,192$ see also Dutch, Netherlands

Honshu 62, 54

horses $23,32-45,110$

Horsfield, Thomas 192

Hsüan Tsang 108

Hui 35

Hundeshagen, George H. 196

Hüsken, Frans 177

Iban 87

Iberians 43, 151

Ibrahim Alfian 62

I-Ching 108-9

Ilocanos $86,88-9$

Ilocos $81-2,84,87-89,91,93-4$

Impey, Oliver 186

Inderagiri 73

India(n)(s) 4-5, 9, 32, 34-9, 44, 47, 51, 57, $62,68,73,101,104-6,108-10,113-4$, $116,122-4,126-7,129,131,134-6,138$, $146,150,153,15^{6-7}, 160-1,164-5$, 188, 192

British 35

Mughal 188

Ocean $36,62,68,106,108,110,113,122,126,129$

Indianization $7,13,123,132$

Indochina $5,42,44,106,121,131$

Indonesia(n)(s) 1-2, 4-13, 38, 43, 62-6, 131, 181, 187, 192, 194, 197-9, 200-2

Eastern 100, 134-5, 137, 146-7, 154, 161, 174

Indrapatra 73

Indrapura 73

Indrapuri 73

Indrapurwa 73 
Islam(ic) $4,7,10,66,73,75,80,121-2,126-7$, $137,144,146,180,186,188,190,198-9$ see also Muslims

Islamization $\quad 132,144$

Jambi $\quad 73,109,151,154-6,158,161$

Jansz. Coeman, Jacob 184

Japan(ese) 13, 23, 29-30, 38, 43, 63-4, 70-1, 104, 130, 147, 165, 185-6, 188

Java(nese) $\quad 1,4-5,7,9-10,35^{-6}, 3^{8-9}, 41-3$, 46-53, 55-6o, 63-6, 69-70, 75-7, 80, 83, 104, 106-7, 109, 112-3, 115-7, 120-3, 126-8, 130-1, 135, 138, 140-2, 145, 149-55, $157-8,161,163-5,171-3,177,184,188-97$, 199, 201

Sea $138,15^{2}$

War 42, 46 see also Diponegoro War

Jeneberang River 140, 143

Jesuits 92

Johor $15^{2}$

Jolo 24

Jordana y Morera, Ramón $\quad 28$

Jörg, Christiaan 186

Kalimantan $\quad 134-5,138,166-7,180$ see also Borneo

Kalla, Jusuf $\quad 177$

Kamchatka 64

Karang Berahi 73

Kathirithamby-Wells, J. $\quad 62,144$

Kayuwangi, Rake $\quad 48,50,52-7,61$

Kedah 157

Kedu 39, 47

Kelantan 45, 154

Kelut, Mt. 63,75

Kerinci 73

Khorat Plateau 33

Kielstra, E.B. 62

Kiritappu 71

Klenteng Sentiong $\quad 189$

Klerck, E.S. de 62

Knaap, Gerrit 153

Kondratiev, Nikolai $\quad 3,120$

Korea 45

Krakatau, Mt. 9, 64-6, 68, 75

Krung Raya 73

Kuamang 151

Kupang 174-5

Kutch 39
Kwee Hui Kian $\quad$ 11-2, 116

Kyaukse 40

Laguna de Bay $\quad 79,82,88$

Lamri $73^{-5}$

Lande, Carl 174

landscapes $182,193,201$

Laos 129

Latin America 80

Leopold, Aldo 16

Lesser Sunda Islands see Nusa Tenggara

Leur, Jacob van $\quad 5,129$

Levi-Strauss, Claude $\quad 3,167$

Leyte $\quad 82,84,92,94$

Lieberman, Victor $\quad 11,103,130-1,137,167$

Ligor $109,115,15^{2-4}$

Lisbon 101

Liu Ch'ang 112

Liu Sung 108

Loarca, Miquel de $\quad 85^{-6}$

Lombard, Denis 62

Lombok 36, 66

longue durée $\quad 1-3,7-10,12-15,31-2,45,78,95$, $100,118,120,125,131-2,166-7,180-1,183$

Loro Jonggrang 53

Los Baňos 22-3

Lubuk Tua 73

Lund, Christian 179

Luoyang 107

Luzon $18,22,25,29,36,56,80-3,88$, 9o, 103,162

Ma Huan 40, 74

Macao 138,158

Maḍang 52-3, 56

Madrid 101

Magelang 56

Magindanao 24

Mahayana Buddhism 9

Majapahit $\quad 41,123,130,198$

Makassar(ese) 11, 121, 133-48, 152-3, 157, 160-1

Malabar $114,116,158$

Malacca 112, 115, 120-1, 127 see also Melaka Straits of 106,127

Malang 51, 66

Malay Peninsula $\quad 37,104,106-7,109-10$, $112,115,117,123,130,138-9,154,156$, $158,163,165$ 


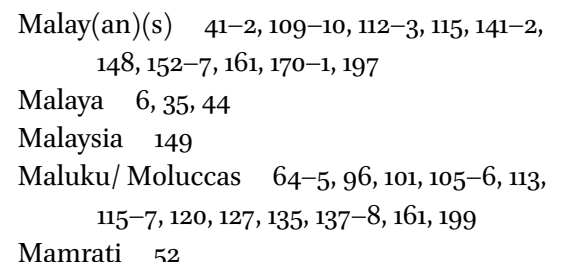

\section{Mamrati 52}

Mandara (Meru), Mt. $\quad 5^{8}$

Mandarin 35

Manguin, P-Y. 129

Manila $\quad 24-8,95,103,138-9,151-2,154,160$ Manila Bay 82, 95

Marcos, Ferdinand 30

Marsden, W. 66

Mataram 76-7, 117, 123, 126

Mathieusen, Jacob 184

Mauritius 36

Maurits, Johan $\quad 185$

McCann, W.R. $\quad 63,71$

Medan 73

Mediterranean $\quad 3,6,101,106,114,121,134-5$

Meilink-Roelofsz, M.A.P. 121

Mekong delta $152,155,164$

Melaka 15, 136, 138-9, 150-4, 16o-2 see also Malacca

Straits of $37,136,139$

Meng Kuan 111

Mentawai $63,65,72$

Mentok 154

Merapi, Mt. $\quad 46-50,56,59,63,75^{-6}$

Merbabu, Mt. $\quad 47-8$

Merkus de Kock, Baron Hendrik 195

Meru, Mt. see Mandara, Mt.

Meulaboh 71

Mexico 103

Middle East(ern) 39, 66, 112-4, 117-8, 122, $146,160,187$

militias $12,166-7,177-80$

Minangkabau $73-4,156-7,163$

Mindanao $16,24,27,29,38,80,103,107$, $117-8,135$
Ming $\quad 74-5,111-2,127$ dynasty 37,113 emperor $\quad 151$

Mohammad Said 62

Mongolia/ Mongols 33-4, 123

Morga, Antonio de $\quad 83,87$

Moro 25, 94

Moulmein 35

Muara Jambi 73

Muara Takus 73

mules $32,34-5,38,42-4$

Mus, Paul 5

Muslims $\quad 35,41,174$ see also Islam(ic)

Naess, Arne 15

Nagcarlan $79,90,93$

Nam-Viet 111-2

Nan Yüeh 105

Nan Zhao 33, 41

Nash, Manning 174

naturalia $11,96-7,101-8,111,115,118$

Neé, Luis 103

Negros 16, 82, 94

Netherlands $\quad 181-2,186,195^{-6}$ see also Dutch, Holland

Netherlands (East) Indies 173, 181,196

New Guinea 37, 117 see also Papua

New Guinea

New Order $12,166-7,176-8$

New Spain 23, 38 see also Americas

New World 103, 175

New Zealand 65

Newcomb, K.R. $\quad 63,71$

Newson, Linda 10

Niai Loro Kidul 10

Nias $63,65,72$

Nieuwenroode, Cornelia van $\quad 185$

Njai Ageng Penatij 198

Njoto 175

Nurudd-din ar-Raniri 74

Nusa Tenggara $9,36,115,15^{2}$

Nusa Tenggara Timor 175

Ottoman Empire $\quad 146$

Owen, Norman $\quad 78-9,86$

Pacific Ocean $\quad 3^{8}$

Padang $\quad 153,161$

Padang Lawas 73

Paduanis, Franciscus de 97

Pagan 130-1

Pagarruyung 73

Pahang 44, $15^{2}$

paintings $12-3,97,101,104,182-6,188,191-2$, 194, 201

Palawan 16 
Palembang $73,109,15^{0-1}, 153^{-4}, 156,15^{8}$, 161-2

Panay 82, 85, 94

Pangandaran 63

Papua New Guinea 64 see also New Guinea

Partai Komunis Indonesia (PKI) 175

Pashtun 45

Pasir 157

Patani 115, 151, 154

Patras, Abraham 190

patronage $153,168,173-9,182$

patron-client $12,168,171,173-4,176-7,179$

Payen, Antoine Auguste Joseph 193

Pegu 40, 101

Pelras, Christian 177

Penang 42

Penanggungan, Mt. $\quad 58,147,154$

Persia(ns) $\quad 38-9,43-4,110,112,114,188$

Persian Gulf 105, 110

Peru 103

Phelan, John 79

Philippines $\quad 6,8-10,15-6,18,20-4,25,27,28$, $30-1,36,38,42-4,56,77-86,88-90,93$, $103,106,115-6,121,127,130,135,149$, $152,174,178$

Spanish $79,81,151-2$

photographs $12-3,182,197-202$

Phra Thong 71

Phuket 71, 153

Pieneman, Nicolaas 194-6

Pikatan, Rake $48,52-3,5^{6}$

Pinchot, Gifford 18, 22

Pineda y Ramírez, Antonio 103

Pinker, Steven 69

Pires, Tomé 40-1

Plaosan Lor 52

Plasencia, Juan de 79

Pliny the Elder 102

Poland 191

Pollock, Sheldon 123

Polo, Marco 74

Poortenaar, Jan Christiaan 194

Popa, Mt. 40

Portugal/ Portuguese $36,38,44,101,104$, 120-1, 134, 138-9, 144, 158, 16o-1

Prague 96, 101

Prahu massif 54

Prahu, Mt. $\quad 46,54,192-3$
Prambanan 48, 53, 76

Priangan 39, 43

Prince of Trunajaya 194

Pronk, Cornelis 186-7

Proudfoot, Ian 66

Pulai 154

Punjab 44

Qin Dynasty $\quad 33,100,108$

Rach, Johannes 189

Raden Saleh 195

Rambahan 73

Red River 41

Rees, Johannes van 190

Reid, Anthony $\quad 131-2,137,139,141,145$, 162, 168

Reyes, Raquel 10-11

Rheede, H.A. 103

Rheen, Theodorus Justinus 190

Rhijne, Willem ten 103

Riau 145, 152, 154, 162

Ricklefs, Merle 77

Riebeeck, Abraham van 38

Riebeeck, Joanna van 190

Ring of Fire 9, 64, 75

Pacific 65

Rios Coronel, Hernando de los 26

Rizal 82

Rumphius, G.E. 103

Rungus 87

Russia 130

Śaivism $\quad 5^{1}$

Salween (river) 35

Samar 82, 84, 91-2, 94

Samudra-Pasai 74

San Agustín, Gaspar de 86

Sangora 152

Sañjaya, Ratu $\quad 47,5^{6-7}$

Schoeman, Karel 190

Schrieke, B.J.O. 4-5

Schulte Nordholt, Henk 12

Scott, James 174

Second World War $\quad 5,29-30$

Semarang 160

Senopati 76

Seville 101

Shan (States) 44 
Shih Ch'ung 107

Shih Fa-Hsien 108

Shiva/ Śiva $\quad 10,51-4,56,5^{8-9}$

Siak 73,152

Siam 106, 109, 112, 115, 121-2, 127, 139, 150-2, 162 see also Thailand

Sibolga 67,73

Simeulue 72,75

Sinabung, Mt. 63

Sind 39

Siṇ̣̣ok, Rake $\quad 48$, 50, 59-61

Śivamaṇạala 58

Smith, Peter 79

Snouck Hurgronje, C. 62

Song 123

China $\quad 126$

dynasty 117,122

emperors 37

era 118

South Africa 43

South Asia(n) 15, 110, 123, 129, 160, 162-3

South China Sea 106, 126, 135

Southeast Asian mainland $\quad 7,135$

Spain/Spaniards/Spanish $\quad 22,24-8,36$, $38,42-4,80,85,89-90,92-3,103-4$ 151,160

Specx, Jacques 185

Spice Islands $\quad 25,130,137-8$

Sri Lanka(n) 38, 129, 188 see also Ceylon

Srivijaya $73,108-9,113-5,117,122,126,130,150$

Steurs, F.V.H.A. de 195

Storck, Abraham $\quad$ 183-4

Straits of Malacca see Malacca, Melaka

Sudomo 175

Suharto $\quad 176-7$

Sukarno $173-4$

Sulawesi(ans) $\quad 36,80,86,88,93,118,134-5$ $137-9,147,154,157,194$

South 11, 36, 70, 143, 145-7, 150, 152-3, $157,161,164,170,177-8$

Sultan Ageng of Banten $\quad 186$

Sultan Agung $\quad 46,76-7$

Sultan Sayfoeddin of Tidore $\quad$ 191, 202

Sulu 24, 38, 106, 117, 135, 152

Sea 80,161

sultanate 155

Sumatra(n) 9-10, 36-7, 42, 62-9, 71-7, 100, $106-10,112,115,117,122-3,15^{0-1}, 156,15^{8}$, 161,163
Sumba $\quad 39,65,135$

Sumbawa $36,39,65,135,164$

Sumbing, Mt. $\quad 5^{6}$

Sunan Giri 198

Sundoro, Mt. $\quad 5^{6}$

Sung (era) $\quad 107-8,111-4$ dynasty 113

Surabaya $147,150,160,198$

Surakarta $\quad 76$

Surapati 191

Surat 157

Sutherland, Heather 11

Swellengrebel, Hendrik 188

Syria 105

T'ang 109, 111-2

Tagalog(s) $\quad 79,81-2,84,86,88-91,93-5$

Talloq $137-8,143,145$

Talloq River 140

Tambora, Mt. $\quad 9,65,68$

Tamil 122, 124, 126

Tamilnadu 37

Tamwlang 59

Tang (era) $\quad 126$ dynasty 123

Tansil, Eddy 175

Tarakan 166

Tembesi $\quad 151$

Thailand/ Thais $\quad 71,117,129-31,104,154$, 163-5 see also Siam

Theophrastus 102

Theravada Buddhist/ Buddhism see Buddhism/ Buddhist

Tibet(an) 9, 32, 34-5, 45

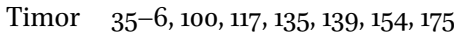

Toemengoeng Soerjawinoto, Raden 198

Tondo $\quad 82,85$

Tonkin 112, 116, 156

Tonle Sap 155

trade/ trading $\quad 6,10-1,24-5,27,34,41,47,74-5$, $80-2,91,96,99,100-2,104-18,120-30$, $134-9,141,144-62,164-5,170,177,186$

Trat $154-5,164$

Trengganu 152, 154, 162

tsunami(s) $\quad 10,63,65,67,69-77,132$

Ungaran $5^{1}$

United States/ USA 27, 29, 44 see also

America, America(ns) 
Untung see Surapati

Utrecht $\quad 188$

Valckenier, Adriaan $\quad 189$

Vienna 101

Vietnam(ese) $7,34,37,39-41,105^{-6,122}$, 129-31, 154, 163, 165

Vihara Buddhayana see Klenteng Sentiong

Vijaya 75

Viljoen, Deon 188

Vinant, Gillis $\quad 182,186$

Visayas, Visayan(s) 24, 36, 79, 80-92, 94

visual history $181,194,200-1$

visual imagery 181

VOC $11,13,25,38,64,103,120-2,128,131$, $136-7,139,141-3,148,15^{2-3}, 155,159-62$, $164,181,184-6,189-91,196$

volcano(es) $9,46-7,51,55^{-7}, 59^{-61}, 70,75^{-6}$

Wade, Geoff $\quad 11,117,122-3$

Wajo(rese) $139,145^{-6}$

Walaṇdit 6o-1

Walraven, Willem 5

Wang Dayuan 74

Wang Gungwu 106
Wawa, Rake $\quad 5^{8-9}$

Wilis, Mt. $\quad 56$

Willem I 194

Willem II 195

Wiraguna, Raden 186

Wisseman Christie, Jan 9, 76, 109, $122-3,140$

Wolters, Oliver $\quad 108,144$

Worcester, D. 29

World War Two see Second World War

Xiamen see Amoy

Yangzi (river) 33-4

Yeh T’ing-kuei 114

Yogya(karta) $\quad 48,63,76,197$

Yuan 126

Yuan dynasty $113,122-3$

Yue $117-8$

Yunnan Plateau $34-5,41$

Yunnan(ese) $\quad 32-5,37,41,45,105$

Zhao Rugua see Chao Ju-kua

Zheng He 74, 112

Zhou Daguan 40 